Для цитирования: Герасимчук М.Ю. Фармакотерапия депрессии: комплексный клинический подход. Сибирский вестник психиатрии и наркологии. 2017; 3 (96): 92-96. https://doi.org/10.26617/1810-3111-2017-3(96)-92-96

\title{
Фармакотерапия депрессии: комплексный клинический подход
}

\section{Герасимчук М.Ю.}

Первый Московский государственный медицинский университет имени И.М. Сеченова Минздрава России (Сеченовский Университет)

Россия, 119991, Москва, ул. Трубеичкая, д. 8, стр. 2

\section{PEЗЮME}

Цель исследования - изучение влияния клинических и хронобиологических характеристик больных на тяжесть депрессии и особенности психофармакотерапии. Материал: проведено клинико-психопатологическое исследование 100 больных депрессией в 2014-2017 гг., из них 68 женщин и 32 мужчины в возрасте от 18 до 77 лет (средний возраст $48 \pm 16$ лет). Методы: клинико-психопатологический, психометрический (MADRS, HDRS-21, PSQI, SPAQ, MEQ, TOB, ИM), статистический. Результаты: В статье описаны результаты изучения влияния клинических и хронобиологических характеристик на особенности психофармакотерапии больных с верифицированным диагнозом депрессии (F31.3-F31.5, F32, F33 по критериям МКБ-10). Заключение. С использованием математических методов статистического анализа определены информативные клинические и хронобиологические факторы, влияющие на тяжесть состояния больного спустя 8 недель терапии: возраст на момент обращения, хронотип, клинический синдром, исходная тяжесть состояния. Индивидуальный биоритмологический статус больного может влиять на эффективность психофармакотерапии. Учет полученных данных может способствовать улучшению качества медицинской помощи данной категории пациентов.

\section{Ключевые слова: психофармакотерапия, антидепрессанты, биологические ритмы.}

\section{ВВЕДЕНИЕ}

Высокая распространенность аффективной патологии определяет социальную значимость заболевания [3, 5, 7]. Наряду с появлением новых антидепрессантов, значимых изменений в эффективности и скорости развития эффекта препаратов не произошло $[6,8]$.

Природа депрессии как «мультифакториального расстройства» (Бохан Н.А., Счастный Е.Д., 2016) [2], интерес к роли мелатонина $[1,16]$, синхронизации физиологических ритмов демонстрируют перспективность поиска биологических диагностических показателей для разработки персонализированного подхода [11]. Так, за последние годы в зарубежной литературе активное внимание уделяется изучению риска развития психической патологии, тяжести депрессии и переносимости фармакологических препаратов у лиц с разным характером суточной активности (хронотип) [4, 10, 12, 14, 17]. Применение информационных технологий в здравоохранении на современном этапе уже на начальной стадии обследования может позволить прогнозировать промежуточный и конечный результаты применения стандартных схем лечения.

Цель исследования - изучение влияния клинических и хронобиологических характеристик больных на тяжесть депрессии и особенности психофармакотерапии.

\section{МАТЕРИАЛЫ И МЕТОДЫ}

Комплексное клинико-психопатологическое исследование проведено при участии 100 больных депрессией, поступивших на лечение в Клинику психиатрии им. С.С. Корсакова Университетской клинической больницы № 3 ФГБОУ ВО Первый МГМУ им. И.М. Сеченова Минздрава России в период 2014-2017 гг.
В исследовательскую выборку вошло 68 женщин и 32 мужчины в возрасте от 18 до 77 лет (средний возраст составил $48 \pm 16$ лет).

Критерии включения: 1) диагноз депрессии, установленный согласно МКБ-10 - депрессивный эпизод, F32 (n=27); рекуррентное депрессивное расстройство, F33 (n=53); биполярное аффективное расстройство, F31.3-31.5 (n=20); 2) добровольное информированное согласие пациентов на участие в исследовании.

Критерии невключения: депрессия в рамках шизофрении, органического поражения ЦНС, болезней зависимостей (алкоголизм, наркомания); беременность, кормление грудью.

Критерии исключения: отказ от дальнейшего участия, тяжелые заболевания внутренних органов

Для достижения поставленной цели использованы следующие методы и подходы: сбор анамнестических данных, клинико-психопатологическое исследование с применением шкалы депрессий Монтгомери-Асберга (MADRS), Питтсбургского опросника для определения качества сна (PSQI), Опросника для оценки сезонного паттерна (SPAQ). Методика для оценки циркадных ритмов человека Morningness Eveningness Questionnaire (MEQ) позволила выделить лиц с вечерним (В), промежуточным (П) и утренним (У) хронотипами. Суточные колебания настроения оценивались по шкале Гамильтона (HDRS-21, пункт 18a), особенности восприятия времени при аффективных расстройствах - по Тесту осознавания времени (ТОВ) и «индивидуальной минуте» (ИМ, по методу Halberg). Для оценки эффективности психофармакотерапии проводилось определение числа респондеров (R), у которых выявлено снижение исходного балла MADRS более чем на $50 \%$ к 8-й неделе терапии. 


\section{РЕЗУЛЬТАТЫ И ОБСУЖДЕНИЕ}

В ходе исследования был проанализирован ряд параметров, отражавших периодические закономерности течения заболевания. Возраст больных на момент начала заболевания варьировал от 15 до 75 лет (в среднем 38,06 $\pm 14,94$ года), длительность заболевания колебалась от 1 месяца до 53 лет 6 [1,5; 13; 5]). За этот период больные перенесли от 0 до 25 эпизодов $(3[0 ; 6])$ без учета настоящего, продолжительностью от 0 до 24 месяцев (3 [2; 4]). Доля повторных эпизодов - 72\%. Средний балл по MEQ

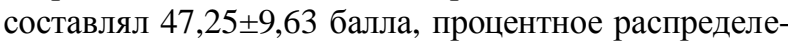
ние по хронотипам: У $-12 \%, \Pi-58 \%, \mathrm{~B}-30 \%$.

Психометрическая оценка по самоопроснику MEQ была оправдана в связи с корреляцией между субъективными и объективными результатами из-

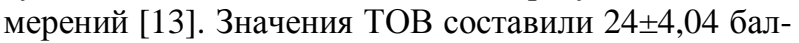
ла, «индивидуальная минута» была значительно

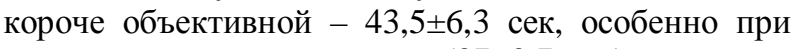
меланхолической депрессии $(37 \pm 3,7$ сек), что отражало нарушение согласованности восприятия времени при аффективных расстройствах.

Сезонный паттерн в соответствии с Опросником SPAQ был установлен в $13 \%$ случаев, с осеннезимней периодичностью у лиц с униполярной депрессией. Примечательно, что параметр сезонности был значимым до начала заболевания. Среди больных с биполярным расстройством пик рождаемости приходился на январь (25\%), при рекуррентном $(\mathrm{n}=53)$ - на июнь (19\%), в случае впервые выявленного эпизода $(\mathrm{n}=27)$ отмечалось два разнонаправленных пика.

В соответствии с Опросником PSQI качество сна - наиболее ранний и очевидный показатель нарушения циркадных ритмов. Данный показатель был ниже при повторных эпизодах в рамках рекуррентного и биполярного расстройств - 17,6 $\pm 5,9$ и 17,5 $\pm 3,5$

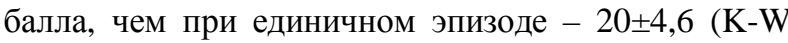
test, $\mathrm{p}=0,013)$; время засыпания: 55 [30; 60], 30 [10; $50]$ и 30 [10;50] соответственно (K-W test, $\mathrm{p}=0,017)$.

В таблице 1 приведены признаки, которые имели значимую степень корреляции с PostMADRS $(\mathrm{p}<0,05)$.

Т а б л и ц а 1

Признаки со значимой степенью корреляции c PostMADRS $(\mathrm{p}<0,05)$

\begin{tabular}{|c|c|}
\hline Признак & $\begin{array}{c}\text { Гамма- } \\
\text { корреляция }\end{array}$ \\
\hline Возраст на момент обращения & 0,141063 \\
\hline Пол & 0,135109 \\
\hline Диагноз & $-0,301927$ \\
\hline Первый/повторный эпизод & 0,358283 \\
\hline Клинический синдром & 0,225734 \\
\hline Хронотип & 0,499231 \\
\hline MEQ & $-0,264373$ \\
\hline Длительность депрессивного эпизода, месяцы & 0,160436 \\
\hline Продолжительность заболевания, годы & 0,242361 \\
\hline PSQI & $-0,144060$ \\
\hline PreMADRS & 0,624713 \\
\hline dMADRS & $-0,675944$ \\
\hline
\end{tabular}

Результаты регрессионного анализа с пошаговым включением переменных представлены в таблице 2.
Т а б л и ц а 2

Результаты регрессионного анализа

с пошаговым включением переменных

\begin{tabular}{|l|c|c|c|c|c|}
\hline & БЕТА & $\begin{array}{c}\text { Ст.Ош. } \\
\text { БЕTA }\end{array}$ & В & $\begin{array}{c}\text { Ст.Ош. } \\
\text { В }\end{array}$ & $\mathrm{p}$ \\
\hline Св. член & & & $-16,2281$ & 3,087306 & 0,000001 \\
\hline Пол & 0,136960 & 0,074555 & 0,9457 & 0,514817 & 0,069364 \\
\hline Возраст & 0,185187 & 0,073807 & 0,0379 & 0,015116 & 0,013816 \\
\hline Хронотип & 0,283523 & 0,082438 & 1,5094 & 0,438890 & 0,000871 \\
\hline Синдром & 0,198092 & 0,070865 & 0,3615 & 0,129329 & 0,006285 \\
\hline РreMADRS & 0,495117 & 0,078067 & 0,7279 & 0,114776 & 0,000000 \\
\hline
\end{tabular}

Из полученных данных видно, что при условии $\mathrm{p}<0,05$ наибольшую степень влияния на PostMADRS оказывали такие факторы, как возраст больного на момент обращения, хронотип, синдром, исходная тяжесть состояния (PreMADRS). В целом модель взаимосвязи отклика с предикторами достаточно адекватная, коэффициент множественной корреляции $\mathrm{R}=0,736$ близок к $1, \mathrm{a}^{2}=0,541$ больше, чем 0,5 , и описывает более $56 \%$ изменчивости отклика.

Суммарный балл MADRS при поступлении и на 8-й неделе у мужчин и женщин не различался: $29,02 \pm 1,9$ и $29,03 \pm 2,8 ; 12,3 \pm 2,9$ и $13,07 \pm 3,5$ (р>0,05).

Балл PostMADRS имел более высокие значения в старших возрастных группах 56-70 лет: 13,47 $[10,1 ; 16,8]$, чем в группе $18-25$ лет - 11,5 [8,8; 14,2] (K-W test; $\mathrm{p}=0,053)$, при поступлении значения PreMADRS были сопоставимы $(29,87$ [28,09; 31,65] и 28,67 [25,9; 31,38], K-W test; p>0,05).

В представленной выборке были выделены меланхолические $(\mathrm{n}=24)$, тревожные $(\mathrm{n}=33)$, анестетические $(\mathrm{n}=11)$, адинамические, апатические $(\mathrm{n}=14)$ и ипохондрические $(\mathrm{n}=18)$ депрессии. Ипохондрический синдром был ассоциирован с более тяжелым состоянием при поступлении и спустя 8 недель: 30,56 балла $[28,7 ; 32,4]$ и 15,33 балла $[12,1 ; 18,6]$, при тревожном - 29 баллов [27; 30,9] и 11,9 балла [8,6; 15,2] (Mann-Whitney test: $\mathrm{p}=0,006, \mathrm{p}=0,002$ ). Закономерно более высоким значениям PreMADRS соответствовало более тяжелое состояние по PostMADRS (Wilcoxon test, $\mathrm{p}<0,0001$ ).

Подбор комплекса лекарственных препаратов производился индивидуально. Схемы купирующей терапии депрессий, применяющиеся в исследовании: антидепрессант в качестве монотерапии (17\%) или в сочетании с нормотимиком (11\%), нейролептиком (45\%), нормотимиком и нейролептиком (27\%). При первом эпизоде доля респондеров была несколько выше - 79\%, чем при повторных эпизодах $-75 \%$ и $63 \%\left(\chi^{2}=2,28, c c=2, p=0,32\right)$. При рекуррентной депрессии монотерапия антидепрессантом по сравнению с другими препаратами была ассоциирована с улучшением к 8-й неделе $\left(\chi^{2}=6,31, \mathrm{cc}=3\right.$, $\mathrm{p}=0,032$ ).

Единственным параметром, включенным в модель, отражавшим биологические закономерности, являлсял хронотип. У больных с вечерним предпочтением (В) тяжесть состояния была выше как исходно, так и спустя 8 недель терапии (K-W test; $\mathrm{p}=0,002 ; \mathrm{p}=0,001)$ (рис. 1). 

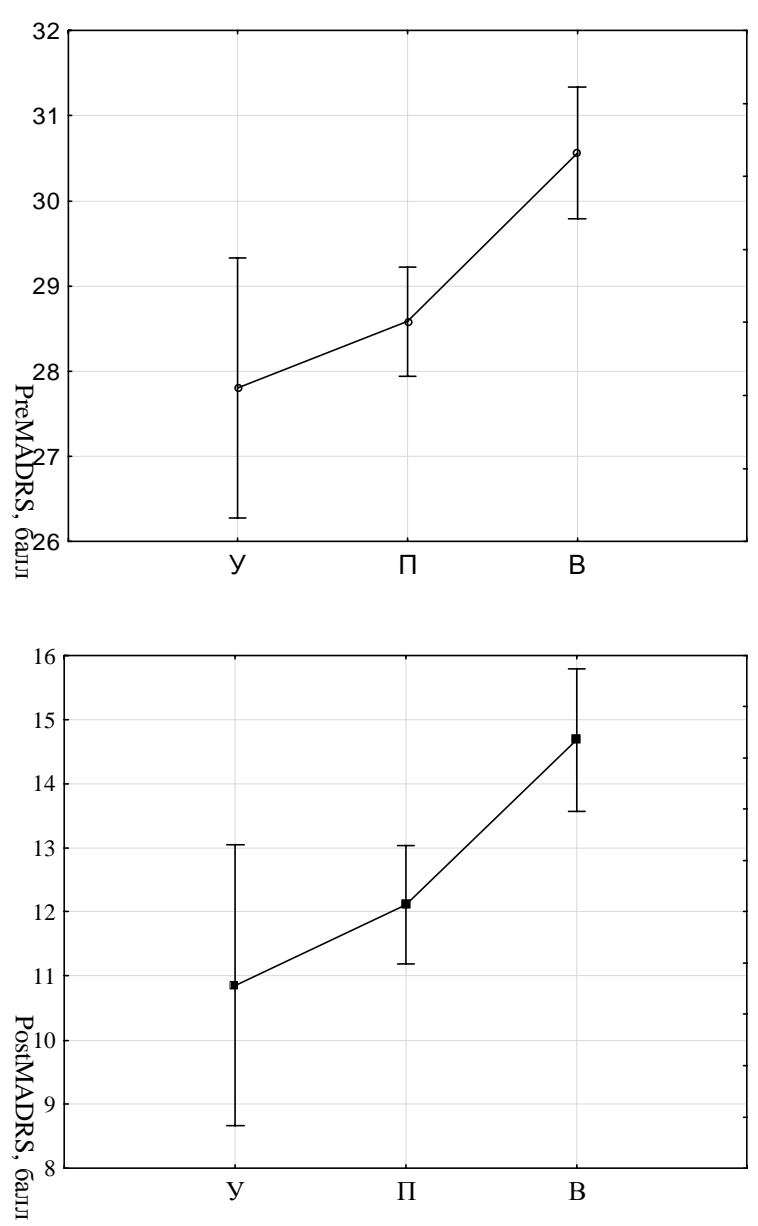

Пр и м е ч а н и е. По оси ординат - суммарный балл MADRS; по оси абсцисс - утренний, промежуточный, вечерний (У, П, В) хронотипы больных

Рисунок 1 - Различия между тяжестью депрессии при поступлении (PreMADRS) и спустя 8 недель терапии (PostMADRS) у больных с различными хронотипами

Анализ распределения больных по числу респондеров показал, что эффективность психофармакотерапии при вечернем хронотипе была выше в случае назначения антидепрессантов (АДП) в первой половине дня (92\%), приема трициклических АДП и селективных ингибиторов обратного захвата серотонина ( $\mathrm{RB}=70 \%, \mathrm{Ry}=33 \%$; $\mathrm{RB}=72 \%, \mathrm{Ry}=50 \%)$. Для больных с утренним хронотипом - во второй половине дня $(52 \%, \mathrm{p}<0,0001)$, при приеме АДП «других» классов (ингибиторов МАО, селективных и полуселективных ингибиторов обратного захвата серотонина и норадреналина, селективных ингибиторов обратного захвата норадреналина, блокаторов серотониновых и адренорецепторов, мелатонинергического препарата) ( $\mathrm{Ry}=100 \%, \mathrm{RB}=54 \%, \mathrm{p}=0,031$ ), АДП с дополнительным седативным (амитриптилин, миртазапин, миансерин, пипофезин, флувоксамин) и сбалансированным действием (мапротилин, пароксетин, пирлиндол, кломипрамин, венлафаксин) $(\mathrm{Ry}=63 \%, \mathrm{R}=43 \%$; $\mathrm{Ry}=100 \%$ и $\mathrm{RB}=64 \%)$.
В целом соотношение респондеров среди больных с разными хронотипами было следующим: $\mathrm{Ry}=75 \%, \mathrm{R}_{\Pi}=73 \%, \mathrm{R}_{\mathrm{B}}=67 \%$. При промежуточном хронотипе показатели занимали средние значения: $\mathrm{R}_{\text {ТЦА }}=73 \%, \quad \mathrm{R}_{\text {СиОзС }}=64 \%, \quad \mathrm{R}_{\text {другие АдП }}=78 \%$. Внутри групп по каждому из хронотипов статистически значимых различий при использовании описанных выше схем терапии не установлено (по числу респондеров, $\mathrm{p}>0,05)$. Установленные закономерности могут быть связаны с клинической картиной заболевания у больных с разными хронотипами и особенностями фармакологического действия препаратов. Так, выявлено преобладание тревожных депрессий при утреннем и промежуточном хронотипах $(58,3 \%)$, меланхолических - при вечернем хронотипе $(30 \%)$. Зарубежными авторами неоднократно было показано снижение доли лиц с утренним типом среди больных по сравнению с популяцией в целом [15] и отмечалась тесная связь между депрессией и вечерним предпочтением [9, 18], исходя из чего последнее может расцениваться как исходно неблагоприятный фактор течения заболевания и прогноза.

\section{ЗАКЮЧЕНИЕ}

По итогам проведенного исследования с использованием математических методов статистического анализа определены наиболее информативные клинические и хронобиологические факторы, оказывающие влияние на тяжесть состояния больного спустя 8 недель терапии: возраст на момент обращения, хронотип, клинический синдром, исходная тяжесть состояния. Полученные результаты указывают на тот факт, что индивидуальный биоритмологический статус больного может влиять на эффективность психофармакотерапии. Учет полученных данных может способствовать улучшению качества медицинской помощи данной категории пациентов.

\section{КОНФЛИКТ ИНТЕРЕСОВ}

Автор заявляет об отсутствии конфликта интересов в связи с публикацией данной статьи.

\section{ИСТОЧНИК ФИНАНСИРОВАНИЯ}

Автор заявляет об отсутствии финансирования при проведении исследования.

\section{СООТВЕТСТВИЕ ПРИНЦИПАМ ЭТИКИ}

Работа соответствует этическим стандартам, разработанным в соответствии с Хельсинской декларацией BMA (протокол заседания этического комитета при ФГАОУ ВО Первый МГМУ им. И.М. Сеченова Минздрава России № 13-14 от 12.10.2014).

\section{ЛИТЕРАТУРА}

1. Алексеева Н.С., Салмина-Хвостова О.И., Белобородова Е.В. Взаимосвязь нарушений пищевого поведения с уровнем мелатонина и серотонина при метаболическом синдроме. Сибирский вестник психиатрии и наркологии. 2016; 4 (93): 39-44.

2. Бохан Т.Г., Бохан Н.А., Счастный Е.Д., Шухлова Ю.А., Галажинская О.Н., Танабасова У.В. Причины и последствия депрессии в восприятии представителей этнических групп Сибири. Современные исследования соииальных проблем (электронный научный журнал). 2016; 9 (65): 47-69.

3. Бохан Т.Г., Терехина О.В., Шухлова Ю.А., Немцев А.В., Галажинская О.Н., Танабасова У.В., Кургак Д.И., Матвеева Н.П., Бадыргы И.О. Особенности стратегий поведения и факторов улучшения состояния у представителей этниче- 
ских групп Сибири с симптомами депрессии. Сибирскии вестник психиатрии и наркологии. 2016; 4 (93):109-116.

4. Герасимчук М.Ю. Хронобиологический паспорт депрессии. Вестник последипломного медицинского образования. 2016; 4: 56-61.

5. Дубровская В.В., Корнетова Е.Г., Микилев Ф.Ф., Дмитриева Е.Г., Мальцева Ю.Л., Корнетов А.Н., Семке А.В. Опыт применения неинвазивной биоимпедансометрии для оценки изменений жировой компоненты состава тела больных шизофренией в ходе антипсихотической терапии. Сибирский вестник психиатрии и наркологии. 2017; 1 (94): 10-14.

6. Семке А. В., Алтынбеков К. С. Фармакоэкономические аспекты антидепрессивной терапии (литературный обзор). Сибирский вестник психиатрии и наркологии. 2014; 1 (82) 71-74.

7. Счастный Е.Д., Симуткин Г.Г., Лебедева Е.В., Яковлева А.Л., Лосенков И.С., Левчук Л.А., Сергиенко Т.Н., Муслимова Э.Ф., Репин А.Н., Нонка Т.Г., Иванова С.А. Клиникобиологические факторы полиморфизма и эффективности терапии аффективных расстройств. Сибирский вестник психиатрии и наркологии. 2016; 4 (93): 26-32.

8. Шацберг А.Ф., Коул Дж.О., ДеБаттиста Ч. Руководство по клинической фармакологии / пер. с англ.; под ред. А.Б. Смулевича, С.В. Иванова. М.: МЕДпресс-информ, 2014 215.

9. Antypa N., Verkuil B., Molendijk M., et al. Associations between chronotypes and psychological vulnerability factors of depression. Chronobiol Int. 2017. Jul. 31: 1-11.

10. Au J., Reece J. The relationship between chronotype and depressive symptoms: A meta-analysis. J. Affect Disord. 2017; 218 93-104.
11. Boiko A.S., Losenkov I.S., Levchuk L.A., et al. Multiplex Approach in Depressive Disorders. Research Opera Med Physiol. 2016; 2 (S1): 33-34.

12. Fischer D., Lombardi D.A., Marucci-Wellman H., Roenneberg T. Chronotypes in the US - Influence of age and sex. PLoS One. 2017; 12 (6): e0178782.

13. Gershon A., Kaufmann C.N., Depp C.A., et al. Subjective versus objective evening chronotypes in bipolar disorder. Affect Disord. 2017; 225: 342-349.

14. Goel N. Genetic Markers of Sleep and Sleepiness. Sleep Med Clin. 2017; 12 (3): 289-299.

15. Müller M.J., Cabanel N., Olschinski C., et al. Chronotypes in patients with nonseasonal depressive disorder: Distribution, stability and association with clinical variables. Chronobiol Int. 2015; 32 (10): 1343-51.

16. Pfeffer M., Korf H.W., Wicht H. Synchronizing effects of melatonin on diurnal and circadian rhythms. Gen Comp Endocrinol. 2017; May 19. pii: S0016-6480(17)30172-7. doi: 10.1016/j.ygcen.2017.05.013. [Epub ahead of print]

17. Taneja R., Hunter K., Burakgazi-Dalkilic E., Carran M. Effect of sleep patterns on levetiracetam induced mood changes. Epilepsy Behav. 2017; Aug 18. pii: S1525-5050(17)30433-X. doi: 10.1016/j.yebeh.2017.07.038. [Epub ahead of print]

18. Watts A.L., Norbury R. Reduced Effective Emotion Regulation in Night Owls. J Biol Rhythms.2017; 32 (4): 369-375.

Поступила в редакцию 11.05.2017 Утверждена к печати 26.06.2017

Герасимчук Мария Юрьевна, аспирант кафедры психиатрии и наркологии.

Герасимчук Мария Юрьевна, mygerasimchuk@gmail.com

УДК 616.89-008.454:616.8-085.2/.3

For citation: Gerasimchuk M.Yu. Pharmacotherapy of depression: a comprehensive clinical approach. Siberian Herald of Psychiatry and Addiction Psychiatry. 2017; 3 (96): 92-96. https://doi.org/10.26617/1810-3111-2017-3(96)-92-96

\section{Pharmacotherapy of depression: a comprehensive clinical approach Gerasimchuk M.Yu.}

I. M. Sechenov First Moscow State Medical University

Trubetskaya Street 8-2, 119991, Moscow, Russian Federation

\section{ABSTRACT}

Objective of study: to study the influence of clinical and chronobiological characteristics of patients on the severity of depression and specifics of psychopharmacotherapy. Materials: A clinical-psychopathological study of 100 patients with depression in 2014-2017 was carried out, of them 68 women and 32 men aged from 18 to 77 years (mean age $48 \pm 16$ years). Methods: clinicalpsychopathological, psychometric (MADRS, HDRS-21, PSQI, SPAQ, MEQ, TAT, IM), statistical. Results: In the article the results of study of the influence of clinical and chronobiological characteristics on the specifics of psychopharmacotherapy of patients with the verified diagnosis of depression (F31.3-F31.5, F32, F33 according to criteria of ICD-10) were described. Conclusion. With the use of mathematical methods of statistical analysis the informative clinical and chronobiological factors were identified, which influenced on the severity of the state of the patient after 8 weeks of the therapy: age to the moment of seeking for help, the chronotype, the clinical syndrome, initial severity of the state. Individual biorhythmological status of the patient can influence the efficiency of psychopharmacotherapy. Taking into account the findings can contribute to the improvement in the quality of the medical aid of this category of patients.

\section{Keywords: psychopharmacotherapy, antidepressants, biological rhythms.}

\section{REFERENCES}

1. Alekseeva N.S., Salmina-Khvostova O.I., Beloborodova E.V. Vzaimosvjaz' narushenij pishhevogo povedenija s urovnem melatonina i serotonina pri metabolicheskom syndrome [Interrelationship of eating disorders and level of melatonin and serotonin in metabolic syndrome] Sibirskij vestnik psihiatrii i narkologii Siberian Herald of Psychiatry and Addiction Psychiatry. 2016; 4 (93): 39-44 (in Russian).
2. Bokhan T.G., Bokhan N.A., Schastnyy E.D., Shukhlova Y.A., Galazhinskaya O.N., Tanabasova U.V. Prichiny I posledstvija depressii v vosprijatii predstavitelejj etnicheskih grupp Sibiri [Causes and consequences of depression in the perception among the representatives of ethnic groups in Siberia]. Sovremennye issledovanija social'nyh problem (jelektronnyj nauchnyj zhurnal) - Modern Research of Social Problems. 2016; 9 (65): 47-69 (in Russian). 
3. Bokhan T.G., Terekhina O.V., Shukhlova Yu. A., Nemtsev A.V., Galazhinskaya O.N., Tanabasova U.V., Kurgak D.I., Matveeva N.P., Badyrgy I.A. Osobennosti strategij povedenija I faktorov uluchshenija sostojanija u predstavitelejj etnicheskih grupp Sibiri s simptomami depressii [Characteristics of strategies of behavior and factors of improvement of the state in the representatives of ethnic groups of Siberia with symptoms of depression]. Sibirskij vestnik psihiatrii i narkologii - Siberian Herald of Psychiatry and Addiction Psychiatry. 2016; 4 (93):109_ 116 (in Russian).

4. Gerasimchuk M.Y. Hronobiologicheskij passport depressii [Chronobiological passport of depression]. Vestnik poslediplomnogo medicinskogo obrazovanija - Postgraduate Medical Education Bulletin. 2016; 4: 56-61 (in Russian).

5. Dubrovskaya V.V., Kornetova E.G., Mikilev F.F., Dmitrieva E.G., Maltseva Yu.L., Kornetov A.N., Semke A.V. Opyt primenenija neinvazivnoj bioimpedansometrii dlja ocenki izmenenij zhirovoj komponenty sostava tela bol'nyh shizofreniej $\mathrm{v}$ hode antipsihoticheskoj terapii [Experience of use of noninvasive bioimpedancometry for assessment of changes of fat component of the body composition of schizophrenic patients in the course of antipsychotic therapy]. Sibirskij vestnik psihiatrii I narkologii Siberian Herald of Psychiatry and Addiction Psychiatry. 2017; 1 (94): 10-14 (in Russian).

6. Semke A.V., Altynbekov K.S. Farmakojekonomicheskie aspekty antidepressivnoj terapii (literaturnyj obzor) [Pharmacoeconomic aspects of antidepressant therapy (literature review)]. Sibirskij vestnik psihiatrii I narkologii - Siberian Herald of Psychiatry and Addiction Psychiatry. 2014; 1 (82):71-74 (in Russian).

7. Schastnyy E.D., Simutkin G.G., Lebedeva E.V., Yakovleva A.L., Losenkov I.S., Levchuk L.A., Sergienko T.N., Muslimova E.F., Repin A.N., Nonka T.G., Ivanova S.A. Klinikobiologicheskie factory polimorfizma i effektivnosti terapii affektivnyh rasstrojstv [Clinical-biological factors of polymorphism and efficiency of the therapy of affective disorders]. Sibirskij vestnik psihiatrii i narkologii - Siberian Herald of Psychiatry and Addiction Psychiatry. 2016; 4 (93): 26-32 (in Russian).
8. Schatzberg A.F., Cole J.O., De Batista C. [Manual of Clinical Psychopharmacology]. A.B. Smulevich, S.V. Ivanov, eds. Moscow, 2014: 215 (in Russian).

9. Antypa N., Verkuil B., Molendijk M., et al. Associations between chronotypes and psychological vulnerability factors of depression. Chronobiol Int. 2017. Jul. 31: 1-11.

10. Au J., Reece J. The relationship between chronotype and depressive symptoms: A meta-analysis. J. Affect Disord. 2017; 218: 93-104.

11. Boiko A.S., Losenkov I.S., Levchuk L.A., et al. Multiplex Approach in Depressive Disorders. Research Opera Med Physiol. 2016; 2 (S1): 33-34.

12. Fischer D., Lombardi D.A., Marucci-Wellman H., Roenneberg T. Chronotypes in the US - Influence of age and sex. PLoS One. 2017; 12 (6): e0178782.

13. Gershon A., Kaufmann C.N., Depp C.A., et al. Subjective versus objective evening chronotypes in bipolar disorder. Affect Disord. 2017; 225: 342-349.

14. Goel N. Genetic Markers of Sleep and Sleepiness. Sleep Med Clin. 2017; 12 (3): 289-299.

15. Müller M.J., Cabanel N., Olschinski C., et al. Chronotypes in patients with nonseasonal depressive disorder: Distribution, stability and association with clinical variables. Chronobiol Int. 2015; 32 (10): 1343-51.

16. Pfeffer M., Korf H.W., Wicht H. Synchronizing effects of melatonin on diurnal and circadian rhythms. Gen Comp Endocrinol. 2017; May 19. pii: S0016-6480(17)30172-7. doi: 10.1016/j.ygcen.2017.05.013. [Epub ahead of print]

17. Taneja R., Hunter K., Burakgazi-Dalkilic E., Carran M. Effect of sleep patterns on levetiracetam induced mood changes. Epilepsy Behav. 2017; Aug 18. pii: S1525-5050(17)30433-X. doi: 10.1016/j.yebeh.2017.07.038. [Epub ahead of print]

18. Watts A.L., Norbury R. Reduced Effective Emotion Regulation in Night Owls. J Biol Rhythms. 2017; 32 (4): 369-375.

Received May 11.2017 Accepted June 26.2017

Gerasimchuk Maria Yu., PhD student, Department of Psychiatry and Addiction Psychiatry, I. M. Sechenov First Moscow State Medical University, Moscow, Russian Federation.

\section{Gerasimchuk Maria Yu., mygerasimchuk@gmail.com}

